\title{
La inversión privada en el Ecuador explicada por la tasa de interés y la inflación.
}

\author{
Private investment in Ecuador can be explained by the interest rate and \\ inflation.
}

Daniel Izquierdo García. ${ }^{1}$, Carlita Mariuxi Erazo Portilla. ${ }^{2}$, José Manuel Gracia Chancay. ${ }^{3,}$ José Castillo Ruano., ${ }^{4}$ Autor ${ }^{5}$ \& Autor ${ }^{6}$

Recibido: 08-03-2017 / Revisado: 08-05-2017 Aceptado: 13-06-2018/ Publicado: 01-07-2018

\begin{abstract}
.
DOI: https://doi.org/10.33262/cienciadigital.v2i3.161

The purpose of this research is to study the influence of interest rates and inflation on private investment in the period 2002-2015 of Ecuador. A compilation of information from different documents related to the subject was made in order to provide useful evidence for the theorization of this model.

The results of the analysis highlight that the interest rate and inflation are key variables that specifically explain the model in the different estimates made, in the MCO model they explain it in $69 \%$, for the logarithmic model in $73 \%$, for the semilogarithmic model log-lin $78.24 \%$, lin-log $70.95 \%$ and in the reciprocal model $68 \%$, in general the variables subject to study have marked very useful conceptions when making decisions in different sectors of the economy in which In some cases, we want to analyze models that imply current market situations. Finally, it was concluded that the interest rate which measures the cost of using money has an inverse relationship with the investment variable, while inflation which takes into account the economic conditions of a country at a given moment has a direct relationship with the purchase of goods that will be used in the future to produce more goods and services.
\end{abstract}

1 Universidad Técnica de Esmeraldas, Extensión la Concordia, Esmeraldas, Ecuador,jones_izquierdo23@hotmail.com

${ }^{2}$ Universidad Técnica de Esmeraldas, Extensión la Concordia, Esmeraldas, Ecuador,

${ }^{3}$ Universidad Técnica de Esmeraldas, Extensión la Concordia, Esmeraldas, Ecuador,

${ }^{4}$ Universidad Técnica de Esmeraldas, Extensión la Concordia, Esmeraldas, Ecuador,

${ }^{5}$ Universidad Técnica de Esmeraldas, Extensión la Concordia, Esmeraldas, Ecuador,

${ }^{6}$ Universidad Técnica de Esmeraldas, Extensión la Concordia, Esmeraldas, Ecuador, 
Keywords: Private Investment, Interest Rate, Inflation

\section{Resumen.}

La presente investigación tiene como objeto estudiar la influencia de las tasas de interés e inflación en la inversión privada en el período 2002-2015 de Ecuador. Se realizó una recopilación de información de distintos documentos relacionados al tema a fin de proporcionar evidencia útil para la teorización de este modelo. Los resultados del análisis resaltan que la tasa de interés y la inflación son variables claves que específicamente explican al modelo en las distintas estimaciones realizadas, en el modelo MCO la explican en un 69\%, para el modelo logarítmico en $73 \%$, para el modelo semilogarítmico log-lin el $78.24 \%$, lin-log el $70.95 \%$ y en el modelo recíproco el 68\%, en general las variables sujetas a estudio han marcado concepciones muy útiles a la hora de la toma de decisiones en distintos sectores de la economía en cuyos casos se desee analizar modelos que impliquen las situaciones actuales del mercado. Finalmente se concluyó que la tasa de interés la cual mide el costo de utilización del dinero tiene una relación inversa con la variable inversión, mientras que la inflación la cual toma en cuenta las condiciones económicas de un país en un momento dado tiene una relación directa con la compra de bienes que se utilizarán en el futuro para producir más bienes y servicios.

Palabras Claves: Inversión Privada, Tasa de Interés, Inflación.

\section{Introducción .}

Desde el estallido de la crisis financiera, el temor está plagando a Ecuador, el temor a una nueva gran inflación. Inevitablemente, la política de tasa de interés cero de los bancos centrales y la inundación del sistema bancario con liquidez en uno La devaluación del dinero, que destruirá los ahorros de los ecuatorianos, advirtió a muchos economistas, Asesores de inversiones y periodistas. Todo esto interesa a los gobiernos sobre endeudados, que son de esta manera proporcionar nuevo alcance financiero. La ansiedad por la inflación elevó el precio del oro e incluso contribuyó a ello.

A través del comportamiento de la tasa de interés se busca explicar, que impacto proporciona la inversión privada del Ecuador, ciclos económicos que serán objeto de estudio en un periodo determinado, que en principio actúa bajo movimientos de las tasas de interés en el sistema financiero nacional a través de las diferentes instituciones financieras

Lo mencionado anteriormente justifica la necesidad de efectuar el presente trabajo, cuya intención es estudiar la tasa de interés para comprender la política que se ha optado en Ecuador, y para ello es necesario conocer la influencia de las tasas de interés en la inversión 
que se realizará a través de la estimación del modelo econométrico tomando en cuenta el periodo comprendido entre 2002- 2015.

La economía del Ecuador, está en un nuevo paradigma de desarrollo basado en la inversión pública, dando un proceso intensivo de promoción de la inversión, con la finalidad de que, ésta, se constituya en una pieza clave del crecimiento del país. Este proceso de promoción se inició con un aumento de la participación del Estado, redefiniendo su papel con mayor participación al sector público, y una variación minina del sector privado. Las variaciones de las tasas de interés que se dan en el Ecuador son motivos para que exista un retroceso en la inversión.

\section{Tasa de interés .}

La tasa de interés según Fabozzi, Modigliani y Ferri es el es el monto cobrado, expresado como un porcentaje del capital, por un prestamista a un prestatario por el uso de los activos. En lo común, las tasas de interés se dan a conocer en periodos anuales, que se visualizan como la tasa porcentual anual. (Fabozzi, Modigliani, \& Ferri, 1996, pág. 214)

En el actual estudio se ha tomado en cuenta la consideración de teorías muy determinante e influyente en la determinación de la tasa de interés: la teoría de Fisher que sustenta la teoría de fondos prestables y la teoría de preferencia de liquidez de Keynes.

\section{Enfoque clásico de Fisher.}

Irving Fisher para plantear su teoría de la tasa de interés hace referencia a dos preguntas:

¿Por qué ahorra la gente? y ¿Por qué piden prestado? enfocado a una economía simplificada, donde los individuos consumen y ahorran su ingreso actual.

Según Fabozzi, Modigliani y Ferri (2007)

"La teoría nos muestra que la postura de ahorrar de los individuos sociales se sostienen gracias a factores que son determinantes y la vez influyen de manera perpetua en las decisiones de los individuos, la tasa marginal de preferencia en el tiempo del individuo, que viene a ser el deseo de cambiar algo del consumo actual para un mayor consumo futuro, la segunda variable es el ingreso; donde con un ingreso mayor, significa que ahorra más, y finalmente la tercera variable es la tasas de interés es decir el interés que los prestatarios pagan por los préstamos, y es lo que hace posible un consumo mayor en el futuro”. 
El equilibrio del mercado es un estado en el cual nadie, desde las entidades económicas, tiene un incentivo para cambiarlo. Refiriéndose a la oferta y la demanda, el punto de equilibrio estará en el punto en el que se encuentran las curvas de demanda y oferta

La teoría de Fisher sustentada en los llamados fondos susceptibles de darse en calidad de préstamo propone que el nivel normal de las tasas de intereses dados está bajo la influencia de la convergencia de dos fuerzas constantes.

Para Fabozzi, Modigliani y Ferri (1996)

Los salarios son la parte principal de los fondos asignados al consumo, que depende de los resultados finales del trabajo colectivo y se distribuye entre los empleados de acuerdo con la cantidad y calidad del trabajo invertido, la contribución laboral real de cada uno y la cantidad de capital invertido. (pág. 219)

En los mercados de la fuerza laboral, los vendedores son empleados de cierta calificación, especialidad, y los compradores son empresas y empresas. El precio del trabajo es el salario básico garantizado en forma de salarios, tarifas, formas de pago a destajo y pago basado en el tiempo. La demanda y el suministro de mano de obra se diferencian por su formación profesional, teniendo en cuenta la demanda de sus consumidores específicos y la propuesta de sus propietarios, es decir, se forman un sistema de mercados para sus tipos individuales. (pág. 219)

\section{Teoría de preferencia de liquidez.}

Según Fabozzi, Modigliani y Ferri (2007)

"La tasa de interés se encuentra profundamente ligada con la teoría de la demanda del dinero expuesta por parte del economista británico J. Keynes. Keynes creía que, en la economía actual, lo más importante es el motivo especulativo para almacenar dinero.

Además, ese dinero es un medio de circulación, se pueden considerar como un activo especial. Bajo el activo se entiende cualquier cosa que sirva para preservar el valor. Cada activo, ya sea una casa, terreno, valores, automóvil o dinero, se caracteriza por dos propiedades: liquidez e ingresos. La liquidez garantiza la facilidad de convertir un activo en otro. El ingreso le da al dueño del activo un sustento.

El dinero es un activo altamente líquido, ya que el dinero en cualquier momento sin pérdida de valor puede convertirse en un bien. El activo que posee más valor 
a partir dl la expresión de la liquidez es el dinero. Aunque no podemos intercambiar valores directamente por bienes y servicios, la disponibilidad de una bolsa de valores -el mercado de valores- hace que sea relativamente fácil vender dichos valores y comprar los productos deseados con los ingresos. Otros activos se venden y compran mucho más difícil. Por lo tanto, si una persona se enfrenta a la opción de dónde invertir, primero comparará el dinero con los valores.

A diferencia de otros activos, el dinero no genera ingresos. La importancia del dinero está solo en su liquidez. Al tener una mínima tasa de interés vigente la disposición en la economía de circulante (dinero) de alta liquidez tiene una gran diferencia, superando por mucho la falta de ingresos que existiese. Al aumentar el porcentaje de interés sobre los valores, la liquidez será poco transcendental que la rentabilidad" (pág. 56).

\section{Inversión.}

Las inversiones son una parte integral de la economía moderna. De los préstamos, las inversiones difieren en el grado de riesgo para el inversionista (prestamista): el préstamo y los intereses deben reembolsarse dentro de los términos acordados independientemente de la rentabilidad del proyecto, las inversiones (capital invertido) se devuelven y rinden ingresos solo en proyectos rentables.

Para Mankiw (2012) "La inversión puede decirse que es la adquisición de bienes materiales que en lo posterior serán usados con el fin de generar más bienes y/o servicios. Es la acumulación de adquisiciones de tipos de capital, inventariado y edificios". (pág. 497)

La inversión proporciona a futuro el dinero que genera diversas actividades económicas. El tiempo ha demostrado que no todo lo que se invierte viene a ser ganancia es por ello que se debe tomar en consideración puntos importante al momento de hacerlo basándose en información de primera fuente, el estado del mercado financiero entre otras que determinen una buena inversión sea a corto o largo plazo.

La tasa de interés es aquel pago que realiza un individuo transfiriéndolo a otra persona, al haberle proporcionado estos fondos a la primera para el uso productivo del circulante prestado. El nivel de interés se da por la relación existente entre las variables de la oferta y la demanda en el mercado de capital de préstamo y se expresa en una tasa de interés, que es la relación entre el valor del interés y el valor del monto del préstamo.

La política de tasas de interés es uno de los instrumentos más importantes y, al mismo tiempo, bastantes complejos para regular las actividades bancarias. Los postulados clásicos 
de la realización e implantación de una escala de medición de tasas de interés deben ser sustentados en el estado en el que se encuentra la demanda y la oferta de recursos que se destinan al crédito, los términos de almacenamiento, el tamaño de los depósitos, las tasas de inflación, etc.

En la neoclásica teoría en económica se postula la existencia de una relación inversamente proporcional entre las variables: tasa de interés e inversión privada, este postulado se basa en los estudios de Oliveira y Texeira (1999), sustentado en los estudios teóricos de Jorgenson (1963) el cual integra variables como "la tasa de interés que mide el costo que se da al poner en utilización el dinero que se tiene en poder" Oliveira y Texeira (1999)también se toma en cuenta la inflación, que da a conocer "las las características económicas de un Estado" Labarca \& Hernandez (2003).

El tamaño de la formación bruta de capital fijo se estima sobre la base del valor total de los activos fijos adquiridos por unidades institucionales (excluyendo los realizados) en el período del informe, así como de un aumento en los activos no procesados que resultaron de las actividades productivas de las unidades institucionales. (Banco Central de Ecuador, 2015).

\section{Materiales y métodos.}

Los Datos correspondientes a la tasa de interés nos

proporcionan las bases de la página web del Banco Central de Ecuador y del Instituto Nacional de Estadísticas y Censos (INEC) correspondientes al periodo 2002-2015.

\section{Datos utilizados en la investigación}

Tabla $\mathbf{N}^{\circ} 1$ : Datos de inversión privada, tasa de interés e inflación

\begin{tabular}{cccc}
\hline Año & $\begin{array}{c}\text { Inversión privada (miles } \\
\text { de USD) }\end{array}$ & $\begin{array}{c}\text { Tasa de interés } \\
(\boldsymbol{\%})\end{array}$ & Inflación $(\boldsymbol{\%})$ \\
\hline 2002 & 7.286 .405 & 15,81 & 12,48 \\
2003 & 7.266 .775 & 13,64 & 7,93 \\
2004 & 7.864 .475 & 9,95 & 1,95 \\
2005 & 8.791 .573 & 9,62 & 2,12 \\
2006 & 9.592 .384 & 9,81 & 3,30 \\
2007 & 8.191 .889 & 10,72 & 2,28 \\
2008 & 9.356 .568 & 9,14 & 8,40 \\
2009 & 8.434 .118 & 9,19 & 5,16 \\
2010 & 9.906 .103 & 8,68 & 3,55 \\
2011 & 10.901 .660 & 8,17 & 4,47 \\
\hline
\end{tabular}




\begin{tabular}{cccc}
\hline 2012 & 12.227 .240 & 8,17 & 5,10 \\
2013 & 12.573 .732 & 8,17 & 2,72 \\
$2014 \mathrm{sd}$ & 13.688 .623 & 8,19 & 3,59 \\
$2015 \mathrm{p}$ & 13.890 .874 & 9,12 & 3,38 \\
\hline
\end{tabular}

Fuente: BCE, INEC

Elaborado por: Los investigadores

Para alcanzar el objetivo de la investigación se implementó un modelo econométrico que busca establecer la relación que existe entre la inversión privada, la tasa de interés y la inflación.

Se utilizó el análisis de regresión de mínimos cuadrados (MCO) ejecutado en el software libre Gretl para obtener los valores correspondientes a las estadísticas que fueron analizadas e interpretadas.

Se estimaron varios modelos entre ellos el lineal, logarítmico, semilogarítmico y recíproco con la finalidad de analizar los valores de sus contrastes y sus gráficos para elegir el modelo que mejor se ajuste a los criterios de contraste existentes en un proceso de análisis econométrico.

\section{Modelo lineal .}

El método de mínimos cuadrados según (Martínez, 2012) es un método de estimación por el cual las cantidades a estimar son determinadas, minimizando la suma de los cuadrados de las diferencias entre los valores observados $Y_{i}$ y los valores estimados $\widehat{Y}_{i}$.

$$
\hat{Y}_{i}=\hat{\beta}_{1}+\hat{\beta}_{2}+\hat{u}_{i}
$$

\section{Modelo logarítmico.}

Lo llamativo del uso del modelo log-log, que hace de este muy popular en su utilización para trabajos empíricos, se da porque la pendiente $\beta 2$ con su coeficiente permite medir la existencia de elasticidad de $Y$ referente de $X$, entonces, una variación en el porcentaje de $Y$ Dado por una mínima variación del porcentaje en $X$. (Gujarati \& Porter, 2010) 


$$
\log \widehat{Y}_{l}=\log \widehat{\beta_{1}}+\log \widehat{\beta_{2}}+\widehat{u}_{i}
$$

\section{Modelo log-lin.}

"En la expresión de este modelado se puede encontrar el crecimiento del porcentaje de $Y$ suscitado por una variación unitaria absoluta de X” (Gujarati \& Porter, 2010).

$$
\log \widehat{Y}_{l}=\widehat{\beta_{1}}+\widehat{\beta_{2}}+\widehat{u}_{i}
$$

\section{Modelo lin- log.}

"En el modelo lin-log se puede hallar un cambio unitario absoluto de Y dado por un cambio en el porcentaje del valor de X" (Gujarati \& Porter, 2010).

$$
\widehat{Y}_{i}=\hat{\beta}_{1}+\log \widehat{\beta_{2}}+\widehat{u}_{i}
$$

\section{Modelo recíproco.}

"Posee la valoración asintótica o el límite que del cuál va a hacerse poseedora una variable explicada, siempre que la variable explicativa $\mathrm{X}$ aumente su valoración de forma indefinida" (Gujarati \& Porter, 2010).

$$
\widehat{Y}_{i}=\hat{\beta}_{1}+\widehat{\beta_{2}}\left(\frac{1}{X_{i}}\right)+\hat{u}_{i}
$$

\section{Discusión y resultados.}

Una vez aplicado los distintos modelos econométricos se procede a elegir el que mejor se ajusta con el criterio de Akaike.

Tabla $\mathbf{N}^{\circ}$ 2: Criterio de Akaike.

\begin{tabular}{cc}
\hline Modelo MCO CORREGIDO & 57.78630 \\
\hline LOG-LOG & 65.13704 \\
LOG-LIN & 63.97409 \\
LIN-LOG & 69.22653 \\
RECIPROCO & 74.71505 \\
\hline
\end{tabular}

Elaborado por: Los investigadores.

Fuente: Gretl. 
Este criterio permite seleccionar el mejor modelo estadístico, para el conjunto dado de datos. Según la tabla anterior escogemos el modelo MCO corregido porque tiene el menor valor en el criterio de Akaike (57.78630).

Modelo de mínimos cuadrados ordinarios.

Variable regresada:

Y= Inversión privada (Ip)

Variable regresora 1:

$\boldsymbol{X}_{\mathbf{1}}=$ Tasa de interés (I)

Variable regresora 2:

$\boldsymbol{X}_{\mathbf{2}}=$ Tasa de inflación $(\mathrm{P})$

Modelo Matemático

$$
\mathrm{Y}=\beta_{0}+\beta_{1} X_{1}+\beta_{2} X_{2}+\mu
$$

\section{Modelo Econométrico}

$$
I p=\beta_{0}+\beta_{1} I+\beta_{2} P+\mu
$$

\section{Modelo de Mínimos Cuadrados Ordinarios}

Tabla N$^{\circ} 3$ Modelo de Mínimos Cuadrados Ordinarios

\begin{tabular}{cccccc}
\hline & Coeficiente & Estadístico t & Valor $\mathbf{p}$ & $\mathbf{R}^{2}$ & $\mathbf{F}$ \\
\hline const & $1,75100 \mathrm{e}+07$ & 8,724 & $2,84 \mathrm{e}-06 * * *$ & & \\
$\mathbf{I}$ & -892050 & $-3,385$ & $0,0061 * * *$ & 0,752851 & 0,000458 \\
$\mathbf{P}$ & 242976 & 0,9836 & 0,3465 & & \\
\hline
\end{tabular}

Elaborado por: Los investigadores

Fuente: Gretl

Estimación del modelo.

$$
I p=17510000-892050 I+242976 \mathrm{P}+\mu
$$


El coeficiente de $I=-892050$, indica la disminución de la inversión privada cuando aumenta la tasa de interés y la inflación se mantiene constante.

El coeficiente de $\mathrm{P}=242976$, indica el aumento de la inversión ante un aumento en un punto porcentual de la tasa de inflación y con la tasa de interés constante.

El valor del estadístico $t$ de $\mathrm{I}=-3,385$ es menor al valor absoluto de $\mathrm{t}=-2$ por lo tanto el coeficiente es significativo.

El valor del estadístico $\mathrm{t}$ de $\mathrm{P}=0,9836$ es menor al valor absoluto de $\mathrm{t}=2$ por lo tanto el coeficiente no es significativo.

El valor de la prueba de significancia de $\mathrm{p}$ valor para $\mathrm{I}=0,0061$ es menor al nivel de significancia de 0,05 lo que significa que es estadísticamente significativo para el modelo.

El valor de la prueba de significancia de $\mathrm{p}$ valor para $\mathrm{P}=0,3465$ es mayor al nivel de significancia de 0,05 lo que significa que no es estadísticamente significativo para el modelo.

$\boldsymbol{R}^{2}=$ Representa la bondad de ajuste del modelo, indicando que el $75,28 \%$ de las variaciones de la Inversión privada (Ip), es explicado por las variables Tasa de Interés (I) y la tasa de inflación (P).

$\boldsymbol{F}=$ Representa la significancia conjunta de los parámetros el cual muestra que el modelo en su conjunto es significativo, ya que a un nivel de confianza del 95\%, el valor P (de F) es menor a 0,05 .

\section{Conclusiones.}

Luego de elaborar un análisis amplio de la inversión privada, la tasa de interés y la inflación en Ecuador, en el período 2002- 2015, y de haber cumplido con el objetivo propuesto en el proyecto de investigación. Se procede a concluir que:

- Al establecer el análisis dentro del periodo de investigación 2002-2015 se ha podido observar que un factor clave que permite el desarrollo del Ecuador han sido las políticas de inversión empleadas, en especial a partir del 2011 tomo fuerza la inversión pública, el cual ha provocado un impacto al redistribuir los recursos hacia los sectores de vulnerabilidad, es así como se ha ido renovando la infraestructura del Ecuador.

- Sin embargo, cabe mencionar que la tasa de interés en Ecuador ha mostrado una tendencia hacia la baja a partir del año 2008, lo cual es resultado de una mayor 
estabilidad del sistema financiero ecuatoriano, debido a la adopción del dólar estadounidense en el año 2000 como moneda de curso legal, dejando atrás el sucre. Asimismo, la tasa de interés en comparación con las economías desarrolladas continúa siendo elevada por lo que no se convierte en un estímulo para la inversión privada.

- De la misma forma, el mejor modelo según el criterio de Akaike es el modelo MCO, los resultados obtenidos en esta estimación econométrica, evidencian que el coeficiente de $\mathrm{I}=-892050$, indica la elasticidad de la inversión privada, esto quiere decir que ante un aumento en un punto porcentual de la tasa de interés y con la tasa de inflación constante, la inversión disminuye.

- Por otro lado, el coeficiente de $\mathrm{P}=242976$, indica que ante un aumento en un punto porcentual de la tasa de inflación y con la tasa de interés constante la inversión aumenta.

- De acuerdo a las teorías expuestas en la investigación, los datos que se obtuvieron con los valores de las variables en Ecuador concuerdan con lo expuesto en la fundamentación teórica que establece una relación inversa entre la tasa de interés y la inversión privada, además se verificó que la inflación es un factor que influye sobre la inversión privada puesto que encarece los costos de los activos no financieros.

\section{Referencias bibliográficas.}

Banco Central de Ecuador. (Diciembre de 2015). Formación bruta de capital fijo. Obtenido de

https://contenido.bce.fin.ec/documentos/PublicacionesNotas/Catalogo/CuentasNacion ales/Anuales/Dolares/FBKFvd.pdf

Fabozzi, F. J., Modigliani, F., \& Ferri, M. G. (1996). Mercados e instituciones financieras. Naucalpan de Juárez, Edo. de México: Prentice-HallHispanoamericana. doi:968-880679-X

Fabozzi, F., Modigliani, F., \& Ferri, M. (2007). Mercados e Instituciones Financieras. México: Prentice-Hall Hispanoamericana S. A.

Gujarati, D., \& Porter, D. (2010). Econometría (Quinta ed.). México D.F: Mc Graw Hill.

Jimbiquiti, R. (2017). Influencia de la tasa de interés en la Inversión Privada en el Ecuador. European Scientific Journal, 201-214.

Jorgenson. (1963). Capital Theory and Investment Behavior. American Economic(2), 247259. 
Labarca, N., \& Hernandez, G. (2003). Determinantes de la Inversión Privada en Venezuela: un Análisis Econométrico para el periodo 1950-2001. Revista de la Facultad de Ciencias Administativas(2), 29-63.

Mankiw, N. G. (2012). Principios de Economía. Ciudad de México: Cengage Learning .

Oliveira, C., \& Texeira, J. (1999). Impacto de la inversión pública sobre la inversión privada en Brasil: 1947-1990. Revista de la CEPAL(67), 71-80. 


\section{Para citar el artículo indexado.}

Izquierdo D., Erazo C., Gracia J. \& Castillo J. . (2018). La inversión privada en el ecuador explicada por la tasa de interés y la inflación. Revista electrónica Ciencia Digital 2(3), 335347.

Recuperado desde:

http://cienciadigital.org/revistacienciadigital2/index.php/CienciaDigital/article/view/161/14 $\underline{1}$

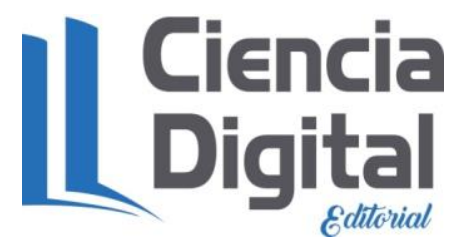

El artículo que se publica es de exclusiva responsabilidad de los autores y no necesariamente reflejan el pensamiento de la Revista Ciencia Digital.

El articulo queda en propiedad de la revista y, por tanto, su publicación parcial y/o total en otro medio tiene que ser autorizado por el director de la Revista Ciencia Digital.
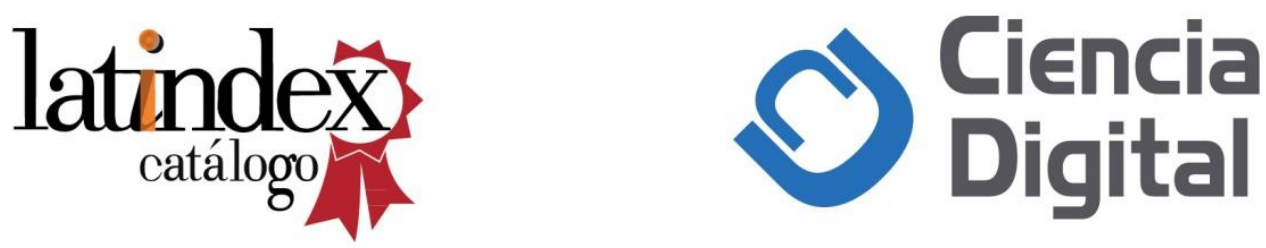